University of South Carolina

Scholar Commons

6-1993

\title{
Accumulation of Maillard Reaction Products in Skin Collagen in Diabetes and Aging
}

Daniel G. Dyer

John A. Dunn

Suzanne R. Thorpe

Karen E. Bailie

Timothy L. Lyons

See next page for additional authors

Follow this and additional works at: https://scholarcommons.sc.edu/chem_facpub

Part of the Chemistry Commons

Publication Info

Published in Journal of Clinical Investigation, Volume 91, Issue 6, 1993, pages 2463-2469.

http://www.the-asci.org/aboutjci.shtml

(C) 1993 by the American Society for Clinical Investigation

This Article is brought to you by the Chemistry and Biochemistry, Department of at Scholar Commons. It has been accepted for inclusion in Faculty Publications by an authorized administrator of Scholar Commons. For more information, please contact digres@mailbox.sc.edu. 


\section{Author(s)}

Daniel G. Dyer, John A. Dunn, Suzanne R. Thorpe, Karen E. Bailie, Timothy L. Lyons, David R. McCance, and John W. Baynes 


\title{
Accumulation of Maillard Reaction Products in Skin Collagen in Diabetes and Aging
}

\author{
Daniel G. Dyer, * John A. Dunn, * Suzanne R. Thorpe, * Karen E. Bailie, ${ }^{*}$ \\ Timothy J. Lyons, ${ }^{\star 8 \|}$ David R. McCance," and John W. Baynes *1 \\ * Department of Chemistry and Biochemistry, and 'School of Medicine, University of South Carolina, Columbia, South Carolina 29208; \\ ${ }^{\ddagger}$ Department of Medicine, Altnagelvin Hospital, Londonderry BT47 1JW', Northern Ireland, United Kingdom; ${ }^{\$}$ Sir George E. Clark \\ Metabolic Unit, Royal Victoria Hospital, Belfast BT12 6BA, Northern Ireland, United Kingdom; "Division of Endocrinology, Diabetes \\ and Metabolism, Medical University of South Carolina, Charleston, South Carolina 29425; and Ralph H. Johnson Department of \\ Veterans Affairs Medical Center, Charleston, South Carolina 29401
}

\begin{abstract}
To investigate the contribution of glycation and oxidation reactions to the modification of insoluble collagen in aging and diabetes, Maillard reaction products were measured in skin collagen from 39 type 1 diabetic patients and 52 nondiabetic control subjects. Compounds studied included fructoselysine (FL), the initial glycation product, and the glycoxidation products, $N^{\epsilon}$-(carboxymethyl)lysine (CML) and pentosidine, formed during later Maillard reactions. Collagen-linked fluorescence was also studied. In nondiabetic subjects, glycation of collagen (FL content) increased only $33 \%$ between 20 and 85 yr of age. In contrast, $\mathrm{CML}$, pentosidine and fluorescence increased fivefold, correlating strongly with age. In diabetic patients, collagen FL was increased threefold compared with nondiabetic subjects, correlating strongly with glycated hemoglobin but not with age. Collagen CML, pentosidine and fluorescence were increased up to twofold in diabetic compared with control patients: this could be explained by the increase in glycation alone, without invoking increased oxidative stress. There were strong correlations among CML, pentosidine and fluorescence in both groups, providing evidence for age-dependent chemical modification of collagen via the Maillard reaction, and acceleration of this process in diabetes. These results support the description of diabetes as a disease characterized by accelerated chemical aging of long-lived tissue proteins. (J. Clin. Invest. 1993. 91:2463-2469.) Key words: aging • collagen • diabetes • glycation • oxidation
\end{abstract}

\section{Introduction}

Glycation (nonenzymatic glycosylation) is a nonenzymatic, posttranslational modification of protein, resulting from chemical reactions between glucose and primary amino groups (1).

Dr. McCance's current address is National Institute of Diabetes and Digestive and Kidney Diseases, 1550 East Indian School Road, Phoenix, AZ 85014.

Address reprint requests to Dr. Lyons, Division of Endocrinology, Diabetes and Metabolism, Medical University of South Carolina, 171 Ashley Avenue, Charleston, SC 29425.

Received for publication 11 May 1992 and in revised form 2 December 1992.

J. Clin. Invest.

(C) The American Society for Clinical Investigation, Inc. $0021-9738 / 93 / 06 / 2463 / 07 \quad \$ 2.00$

Volume 91, June 1993, 2463-2469
The major product in tissue proteins is fructoselysine (FL), ${ }^{1}$ formed by glycation of $\epsilon$-amino groups on lysine residues (Fig. 1). The extent of glycation of tissue proteins depends on the ambient glucose concentration and is relatively constant with age (2-4). However, products formed from FL in subsequent Maillard or browning reactions accumulate gradually with advancing age in long-lived tissue proteins $(2-4)$. These products include $N^{\epsilon}$-(carboxymethyl)lysine (CML) and $N^{\epsilon}$-(carboxymethyl)hydroxylysine (CMhL), which are formed by oxidative cleavage of FL and glycated hydroxylysine respectively (3, 4 ), pentosidine, a fluorescent crosslink formed between arginine and lysine residues (5-7) (Fig. 1), as well as unidentified compounds with characteristic Maillard-type, visible wavelength fluorescence (maxima at $\mathrm{Ex}=328 \mathrm{~nm}, \mathrm{Em}=378 \mathrm{~nm}$ ) (4). CML, CMhL, and pentosidine have been termed "glycoxidation" products $(4,8)$ because both glycation and free radical oxidation reactions are required for their formation from reducing sugars. The exact origin of these compounds, i.e., from glucose vs. ribose $(5)$, ascorbate $(6,7,9)$, or other sugars $(8)$, is uncertain, although present evidence suggests that they are derived exclusively from reactions between proteins and carbohydrates (8).

According to the "glycation hypothesis" of aging and the pathogenesis of diabetic complications, accumulation of endstage products of the Maillard reaction (also known as advanced glycosylation end-products [AGE products]) alters the structural properties of tissue proteins and reduces their susceptibility to catabolism $(10,11)$. These changes contribute to the aging of tissues, and when accelerated by hyperglycemia, to the gradual development of diabetic complications. Despite the attractiveness of this hypothesis, the only Maillard products known to accumulate with age, i.e., CML, CMhL, and pentosidine, result not from glycation alone: oxidation reactions are also necessary for their formation $(2-4,7,11)$. The development of increased fluorescence of proteins in diabetes and aging is also enhanced by oxidation reactions, and carbohydrateor lipid-dependent processes may also be involved (8). These considerations raise questions about the relative importance of the glycation and oxidation components of glycoxidation in the accumulation of Maillard products in tissue proteins.

To our knowledge, no previous studies have made direct comparisons between the concentrations and relative rates of accumulation of the glycoxidation products CML and pentosidine in collagen from control and diabetic populations. There is a similar lack of information on the relative contributions of

1. Abbreviations used in this paper: $\mathrm{CMhL}, N^{\epsilon}$-(carboxymethyl)hydroxylysine; CML, $N^{\epsilon}$-( carboxymethyl) lysine; FL, fructoselysine; $\mathrm{Hb}$, hemoglobin. 


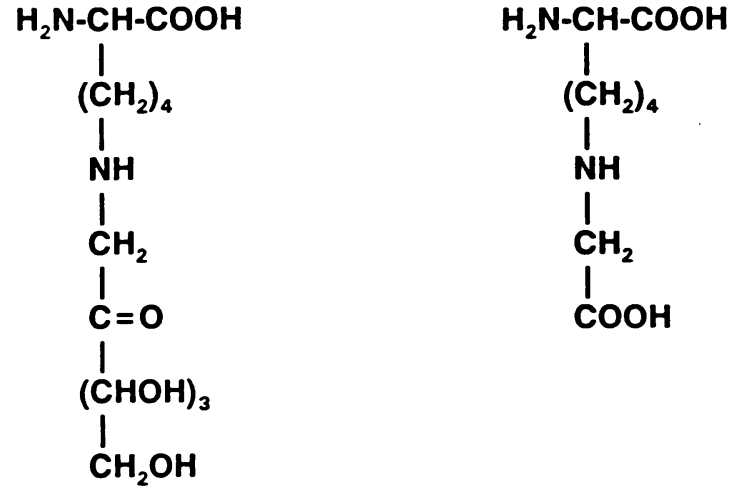

Fructoselysine

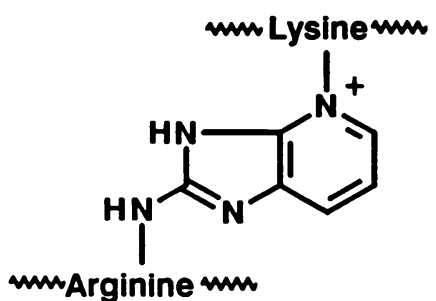

Figure 1. Structures of FL, CML, and pentosidine. FL is the initial product of glycation of protein. The glycoxidation products, CML and pentosidine, are formed by sequential glycation and oxidation reactions between reducing sugars and protein.

\section{Pentosidine}

increased glycation vs. increased oxidation in the accumulation of these products. We have attempted to address these issues in the present study, and show that concentrations of CML and pentosidine, and Maillard-type fluorescence, increase in concert in human skin collagen with age, and that the rate of their increase is accelerated, again in a concerted fashion, in diabetes. Our data also suggest that the accelerated accumulation of glycoxidation products in diabetes can be explained by hyperglycemia and increased glycation alone, without invoking an increase in oxidative stress.

Our findings support the description of diabetes as a disease characterized by accelerated chemical modification and aging of collagen via the Maillard reaction. The relationships between concentrations of these biomarkers and the presence and severity of complications in diabetes are considered in the following article (12).

\section{Methods}

Diabetic patients and control subjects. A total of 91 people were enrolled in this study: 39 patients ( 27 male, 12 female) with insulin-dependent (type 1) diabetes mellitus, aged 17-73 yr (41.1 $15.3 \mathrm{yr}$, mean $\pm S D)$, duration of diabetes $0-46 \mathrm{yr}(17.9 \pm 11.5 \mathrm{yr})$, and 52 nondiabetic control subjects, aged $0-85$ yr $(47.6 \pm 25.9 \mathrm{yr})$. The nondiabetic control skin samples were obtained from healthy, nondiabetic donors, or at autopsy at the Royal Victoria Hospital in Belfast, Northern Ireland. Autopsy samples were taken within $6 \mathrm{~h}$ of death from patients who had died of acute illness, e.g., stillbirth, severe congenital heart disease, trauma, or myocardial infarction. These patients had no history of diabetes and, where possible, had documented normal plasma glucose levels during their terminal illnesses. Some analytical data on the control patients (skin collagen FL and CML concentrations) have been reported previously (3). Live participants in each group were healthy and free of ailments known to elicit inflammatory responses, such as infection, the inflammatory arthritides, or hemochromatosis. Clinical characterization of the diabetic patients and their complication status are presented in the following article (12).

Skin samples. An elliptical full-thickness skin biopsy $(\sim 0.5 \times 1.0$ $\mathrm{cm}$ ) was taken from the medial aspect of the buttock (under $2 \%$ lidocaine local anesthesia in live donors). For $\sim 15 \%$ of the samples, insufficient collagen was available to perform all assays. Thus, the number of samples in some analyses may not always be equal to the number of people involved in the study. Informed consent was obtained from all participants, and the study was approved by the Ethical and Human Subjects Committees of the participating institutions.
Materials. Unless otherwise indicated, reagents were of the highest quality obtainable from Sigma Chemical Co. (St. Louis, MO). The preparation of FL, CML, and pentosidine standards have been described previously $(3,7)$.

Analytical procedures. Procedures for sample storage, for isolation of insoluble collagen by extraction with $1 \mathrm{M} \mathrm{NaCl}, 0.5 \mathrm{M}$ acetic acid, and organic solvents, and for acid hydrolysis of the collagen have been described previously ( 3 ). FL, CML, and lysine in collagen hydrolysates were measured as their $N$-trifluoroacetyl methyl esters by selected ion monitoring gas chromatography - mass spectrometry, as described previously (3). Amounts of FL and CML were normalized to the lysine content of the collagen by external standardization (13) using calibration curves generated from solutions with constant lysine content and variable concentrations of the other analytes. Samples were analyzed in random order without knowledge of the sample identity. Pentosidine was measured in collagen hydrolysates by reversed-phase HPLC using postcolumn fluorescence detection $(E x=328 \mathrm{~nm}, \mathrm{Em}=378 \mathrm{~nm})$, as described previously (7). The assay was standardized with a preparation of pentosidine labeled with $\left[4,5-{ }^{3} \mathrm{H}\right]$ lysine of known specific radioactivity (7). Collagen-linked fluorescence was measured in collagen solubilized by digestion with $2 \%$ pepsin in $0.5 \mathrm{M}$ acetic acid for $24 \mathrm{~h}$ at $37^{\circ} \mathrm{C}$. Fluorescence was measured at both $\mathrm{Ex}=328 \mathrm{~nm}, \mathrm{Em}=378 \mathrm{~nm}$ and $\mathrm{Ex}=370 \mathrm{~nm}, \mathrm{Em}=440 \mathrm{~nm}$, and normalized to the hydroxyproline content of collagen, measured according to Stegemann and Stalder (14), as modified by Maekawa et al. (15). Hemoglobin ( $\mathrm{Hb}$ ) $\mathrm{A}_{1}$ was measured by agar gel electrophoresis (Corning Medical, Halsted, England) after saline incubation to remove labile hemoglobin (16). In the 39 diabetic patients, $\mathrm{HbA}_{1}$ (mean \pm 1 standard deviation [SD]) was $11.4 \pm 2.4 \%$, range $6.9-16.9 \%$. In 24 of these patients, $\mathrm{HbA}_{1}$ values had been obtained every 3 mo for 6 yr preceding the skin biopsy, as described (12). From these results, a mean $\mathrm{HbA}_{1}\left(\mathrm{MHbA}_{1}\right)$ was determined for each patient, and this correlated with recent $\mathrm{HbA}_{1}(r=0.78$, $P<0.0001)$. The normal laboratory range for $\mathrm{HbA}_{1}$ in nondiabetic subjects (mean $\pm 2 \mathrm{SD}$ ) is $3.6-7.2 \%$.

Statistical analysis. Data are shown as means \pm 1 SD. Correlation analyses were performed using the linear least squares method. Statistical data are summarized in figure legends.

\section{Results}

Glycation of collagen. In a previous study of the nondiabetic subjects (3), a slight, but statistically significant, increase in the extent of glycation (FL content) of skin collagen with age was observed. As shown by the lower line in Fig. 2 (from reference 3 , Fig. 4), this amounted to a $33 \%$ increase in glycation of lysine residues in skin collagen between the ages of 20 and $85 \mathrm{yr}$ 


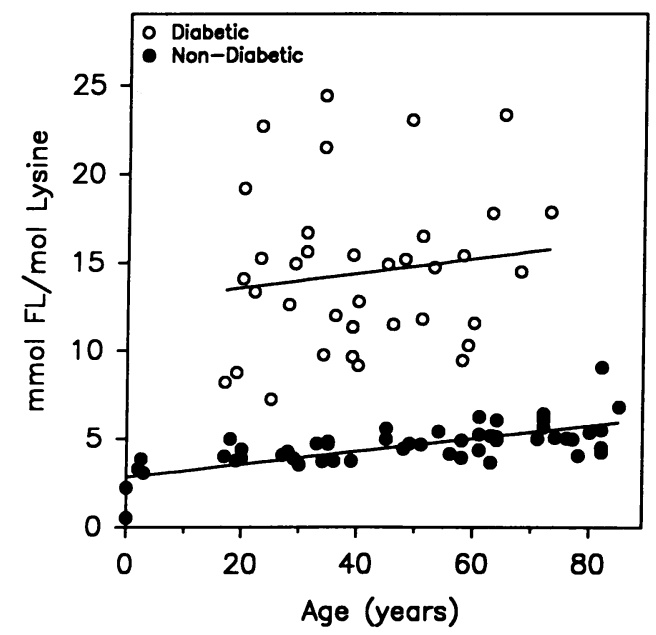

Figure 2. Effects of age and diabetes on glycation of human skin collagen. The equation of the line for control subjects aged $>20 \mathrm{yr}$ (not shown $)$ is: $\mathrm{mmol} \mathrm{FL} / \mathrm{mol} \mathrm{Lys}=[0.029 \times$ age $(\mathrm{yr})]+3.27,(r=0.54$, $P<0.001)$. There was no significant relationship between age and skin collagen glycation in the diabetic patients.

$(r=0.55, P<0.001)$. The upper line in Fig. 2 illustrates that glycation of collagen from the diabetic patients was increased approximately threefold compared with collagen from the nondiabetic control subjects. In the diabetic group, collagen glycation did not correlate either with age or duration of diabetes $(P$ $>0.05$ ). There was a significant correlation between collagen glycation and $\mathrm{HbA}_{1}$ in these patients $(r=0.74, P<0.0001, n$ $=37$ ), confirming the results of previous studies $(17,18)$ : this correlation was strengthened when $\mathrm{MHbA}_{1}$ was used in the analysis $(r=0.89, P<0.0001, n=23)$ (Fig. 3 ).

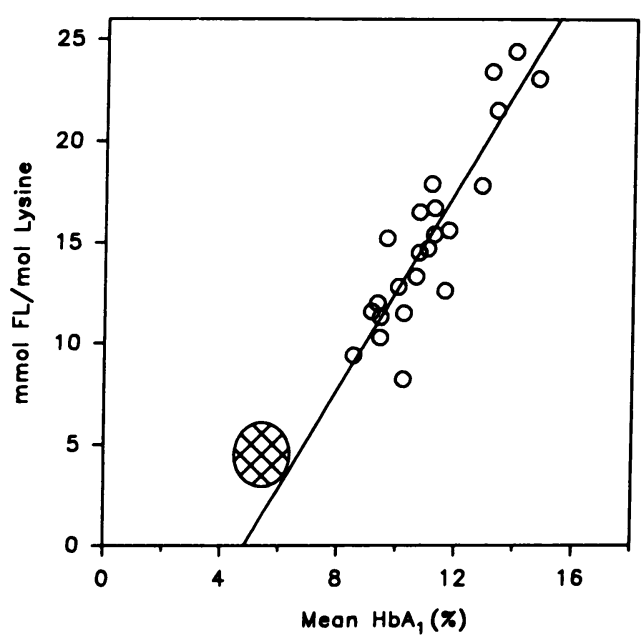

Figure 3. Skin collagen glycation (FL content) in the diabetic population correlates strongly with mean percent glycation of hemoglobin $\left(\mathrm{MHbA}_{1}\right)$, an index of glycemic control over $6 \mathrm{yr}$ before skin biopsy ( $r=0.89, P<0.0001, n=23$ ). The ellipse in the lower left hand corner of the graph indicates the means $( \pm 1 \mathrm{SD})$ of measurements of glycation of collagen (Fig. 2) and percent $\mathrm{HbA}_{1}$ from adult nondiabetic control subjects. Control $\mathrm{HbA}_{1}$ values were obtained from the laboratory reference range, derived from results for 200 nondiabetic subjects.

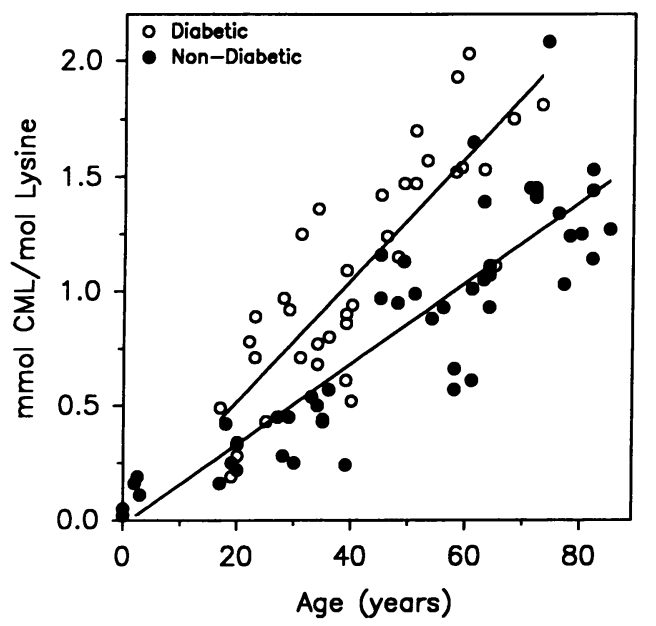

Figure 4. Effects of age and diabetes on the concentration of CML in human skin collagen. The equation of the line for control subjects is: $\mathrm{mmol} \mathrm{CML} / \mathrm{mol}$ Lys $=[0.018 \times$ age $]-0.022(r=0.89, P$ $<0.001)$; and for the diabetic patients: $\mathrm{mmol} \mathrm{CML} / \mathrm{mol} \mathrm{Lys}$ $=[0.027 \times$ age $]-0.015(r=0.84, P<0.001)$.

Glycoxidation products and fluorescence. In previous studies, we have shown that, in contrast to $\mathrm{FL}$, there is a substantial age-dependent increase in the concentration of CML in skin collagen from control subjects (3), i.e., about a fivefold increase between the ages of 20 and $80 \mathrm{yr}$. As shown in Fig. 4, the concentration of $\mathrm{CML}$ also increases with age in collagen from diabetic subjects, but levels of CML in collagen from diabetic subjects are significantly greater than those found in nondiabetic subjects. Analyses for pentosidine (Fig. 5) and fluorescence (Fig. 6) also showed substantial age-dependent increases, with higher levels in collagen from diabetic, compared with age-matched nondiabetic, subjects. To determine the signifcance of the differences between the two groups, each measurement was expressed as a ratio of the observed value to the

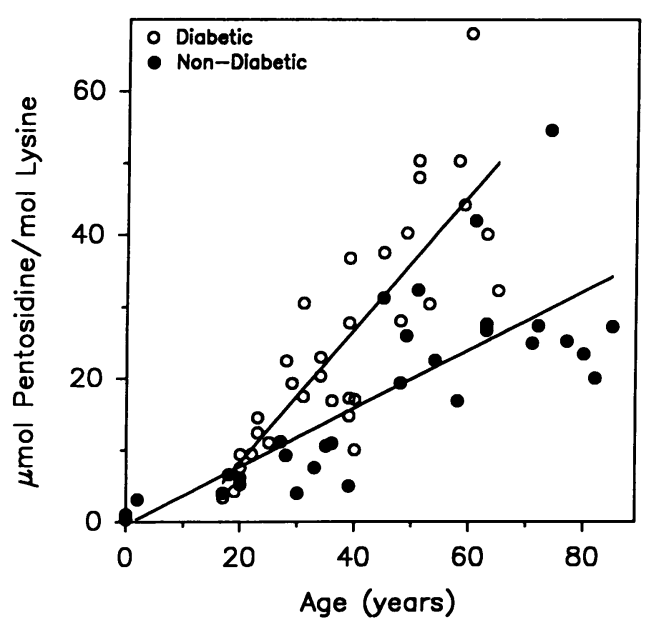

Figure 5. Effects of age and diabetes on the concentration of pentosidine in human skin collagen. The equation of the line for control subjects is: $\mu \mathrm{mol}$ pentosidine $/ \mathrm{mol}$ Lys $=[0.41 \times$ age $]-0.48(r$ $=0.78, P<0.001)$; and for the diabetic patients: $\mu$ mol pentosidine $/$ mol Lys $=[0.93 \times$ age $]-10.27(r=0.83, P<0.001)$. 


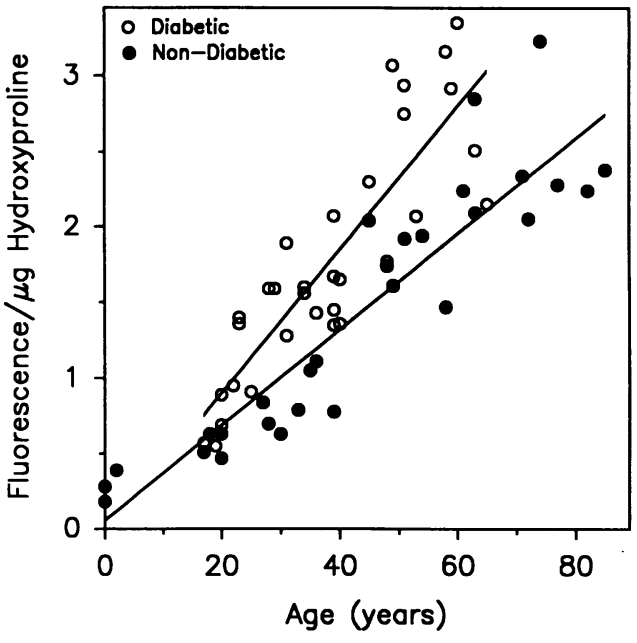

Figure 6. Effects of age and diabetes on Maillard-type fluorescence $(E x=328 \mathrm{~nm}, E m=378 \mathrm{~nm})$ in human skin collagen. The equation of the line for the control subjects is: fluorescence units/ $\mu \mathrm{g}$ Hyp $=[0.032 \times$ age $]+0.056(r=0.92, P<0.001)$; and for the diabetic patients: fluorescence units $/ \mu \mathrm{g}$ Hyp $=[0.047 \times$ age $]-0.047(r$ $=0.86, P<0.001$ ).

predicted value for a control subject of the same age, obtained from the regression line for the control population (Figs. 4-6). By these analyses ( Fig. 7), the control and diabetic populations differed significantly in age-corrected levels of FL, CML, pentosidine, and fluorescence in skin collagen $(P<0.001)$. In contrast to FL, concentrations of CML and pentosidine, and also fluorescence, correlated significantly with duration of diabetes (CML $r=0.74$; pentosidine $r=0.77$; fluorescence $r$ $=0.71, P<0.001)$; these correlations persisted to varying degrees when the concentrations were standardized for age $\left(\mathrm{CML}^{\mathrm{R}} r=0.34, P<0.05\right.$; pentosidine ${ }^{\mathrm{R}} r=0.75, P<0.001$; fluorescence ${ }^{\mathrm{R}} r=0.58, P<0.01$ ).

The strong correlations among the various Maillard products in the control and diabetic populations are summarized in Fig. 8. The correlations between fluorescence and pentosidine in both groups (Fig. 8, top panel) are consistent with the observation that pentosidine accounts for $\sim 30 \%$ of the fluorescence in collagen at $\mathrm{Ex}=328 \mathrm{~nm}, \mathrm{Em}=378 \mathrm{~nm}(4,7)$. There were equally strong correlations between levels of the two glycoxidation products, CML and pentosidine, in collagen from both the control and diabetic groups (Fig. 8, bottom panel). Similarly, the correlations between CML and fluorescence (graph not shown) were highly significant for both groups (control $r$ $=0.94, P<0.001$; diabetic $r=0.95, P<0.001$ ).

Choice of fluorescence maxima. In many reports on "Maillard-type" fluorescence in proteins, measurements are conducted at excitation and emission wavelengths of 370 and 440 $\mathrm{nm}$, respectively. The recent work identifying pentosidine as a major Maillard reaction fluorophore $(5,7)$ and three-dimensional fluorescence spectra of proteins browned by glucose $(4$, 8 ) both suggest that the fluorescence characteristic of the Maillard reaction has excitation and emission maxima close to the maxima for pentosidine, i.e., 328 and $378 \mathrm{~nm}$, respectively. As illustrated in Fig. 9, there is a strong correlation between fluorescence of collagen at $\mathrm{Ex} / \mathrm{Em}=370 / 440$ and that at $\mathrm{Ex} / \mathrm{Em}$ $=328 / 378 \mathrm{~nm}$. However, the fluorescence intensity is about twice as great at $\mathrm{Ex} / \mathrm{Em}=328 / 378 \mathrm{~nm}$ as at $\mathrm{Ex} / \mathrm{Em}=370$ / $440 \mathrm{~nm}$, providing a twofold increase in sensitivity for measurement of Maillard-type fluorescence in collagen if these wavelengths are used.

\section{Discussion}

Glycation of protein in diabetes and aging. The present data show that glycation of collagen (FL content) is increased about threefold in type I, insulin-dependent diabetic patients compared with age-matched nondiabetic control subjects. Glycation of skin collagen correlated with glycation of hemoglobin. However, the correlation was stronger between collagen glycation and $\mathrm{MHbA}_{1}$, an index of average glycemia over a 6 -yr period. This provides new evidence that collagen FL may reflect glycemic control over a longer period of time than $\mathrm{HbA}_{1}$, supporting the conclusions of an earlier study (19). In that study, we observed that the FL content of collagen in type 1 diabetic patients can be decreased by a relatively short $(4 \mathrm{mo})$ period of improved glycemic control, but the proportional decrease in glycation was not as great for collagen as for hemoglobin. In contrast, levels of glycoxidation products and fluores-

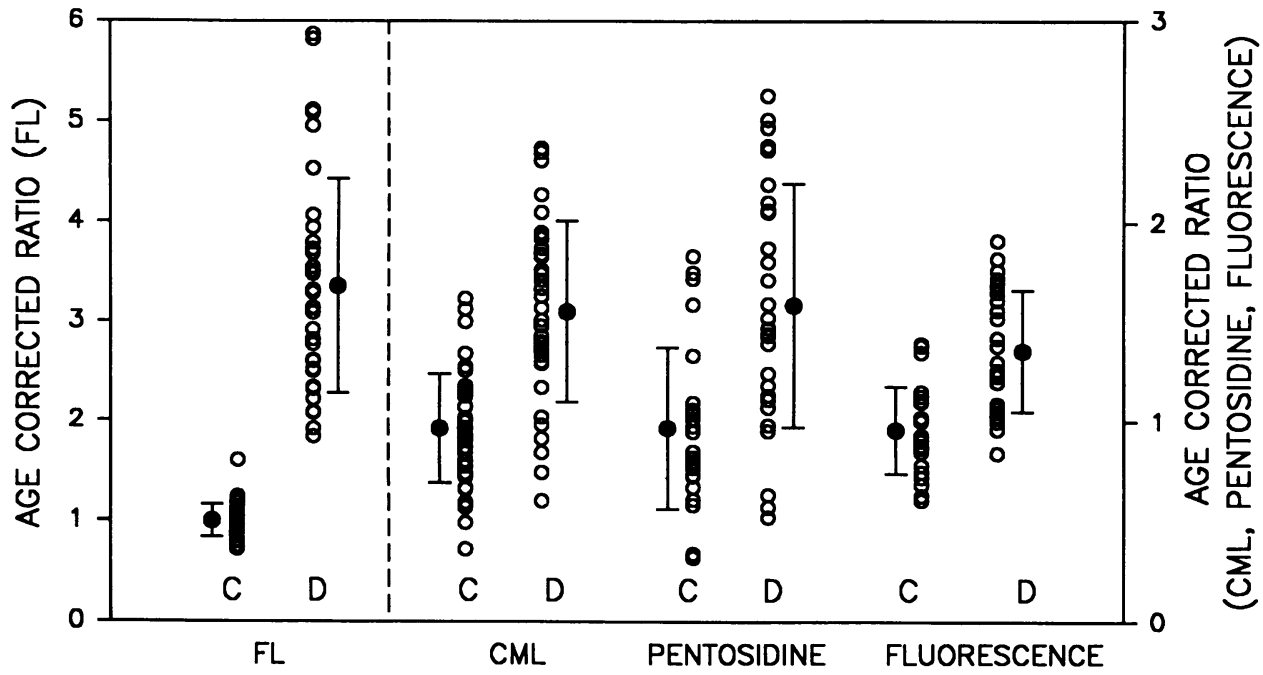

Figure 7. Comparison of age-normalized levels of FL, CML, pentosidine, and fluorescence in skin collagen from control subjects $(C)$ and diabetic pattients $(D)$. The observed value of each parameter for each donor was divided by the calculated value, based on the regression equation for the control population (shown in the legends to Figs. 4-6), then expressed as the ratio of observed to calculated value. Means \pm 1 SD of the ratios are shown. 


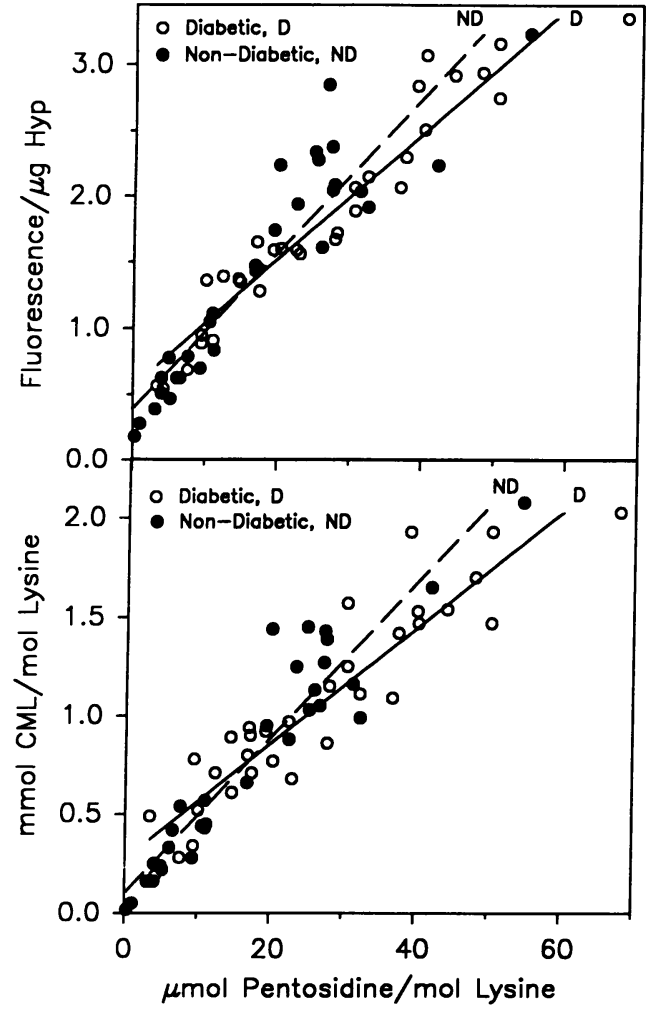

Figure 8. Correlations of fluorescence (top) and CML (bottom) with pentosidine in skin collagen from the control and diabetic groups. Correlation coefficients and $P$ values are as follows: top panel, for the control subjects, fluorescence units $/ \mu \mathrm{g}$ Hyp $=[0.059 \times(\mu \mathrm{mol}$ pentosidine $/ \mathrm{mol}$ Lys $)]+0.39(r=0.92, P<0.001)$, and for the diabetic patients, fluorescence units $/ \mu \mathrm{g} \mathrm{Hyp}=[0.048 \times(\mu$ mol pentosidine $/$ mol Lys $)]+0.57(r=0.96, P<0.001) ;$ bottom panel, for the control subjects, $\mathrm{mmol} \mathrm{CML} / \mathrm{mol}$ Lys $=[0.039 \times(\mu \mathrm{mol}$ pentosidine $/ \mathrm{mol}$ Lys $)]+0.10(r=0.94, P<0.001)$, and for the diabetic patients, $\mathrm{mmol} \mathrm{CML} / \mathrm{mol} \mathrm{Lys}=[0.029 \times(\mu \mathrm{mol}$ pentosidine $/ \mathrm{mol} \mathrm{Lys})]$ $+0.27(r=0.92, P<0.001)$.

cence were unaffected by improved glycemic control. In keeping with the conclusion that collagen FL levels are primarily determined by medium-term (several months [19]) average glycemia, our data demonstrate that, again in contrast to glycoxidation products or fluorescence, Amadori adducts increase only slightly with age in both control and diabetic populations.

Changes in glycoxidation (Maillard) products and fluorescence. Comparative analyses of CML, pentosidine, and Maillard-type fluorescence in skin collagen (Figs. 4-6) indicate that both glycoxidation and fluorescence are increased in collagen from diabetic patients, compared with age-matched nondiabetic controls. The age-normalized data in Fig. 7 also indicate significant differences between the diabetic and control populations in all parameters, independent of age. These results suggest that the increased accumulation of pentosidine (as well as CML and fluorescence) is not confined to a subset of diabetic patients with renal complications, as has been suggested (11), but rather that it is a general feature of the disease process. The comparisons in Fig. 8 emphasize the strong correlations among these three parameters of chemical damage to collagen. The fact that CML and pentosidine are so dissimilar in their structure and physical properties, yet increase in concert both with age and in diabetes, emphasizes their common origin from

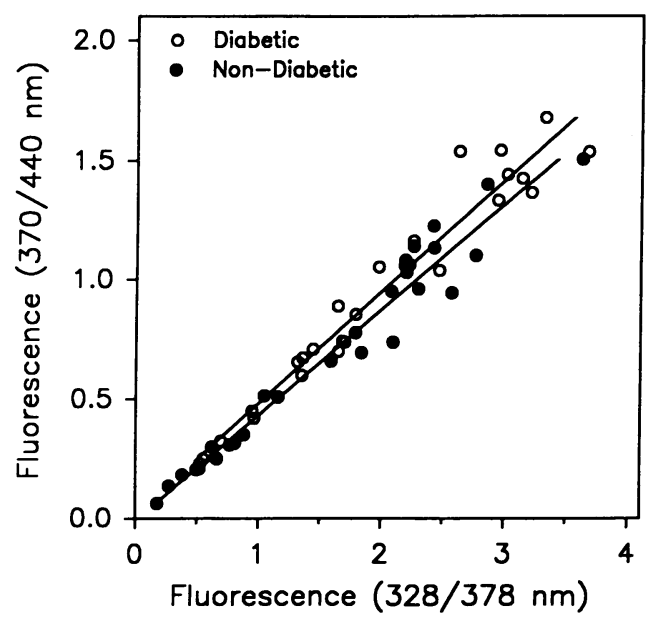

Figure 9. Fluorescence in skin collagen at $\mathrm{Ex}=328 \mathrm{~nm}, \mathrm{Em}=378$ $\mathrm{nm}$, compared to $\mathrm{Ex}=370 \mathrm{~nm}, \mathrm{Em}=440 \mathrm{~nm}$.

glycoxidation reactions. Because the age-dependent rate of chemical modification of skin collagen, as measured by the accumulation of glycoxidation products, is accelerated in the diabetic patients, diabetes may be properly described as a disease characterized, at the chemical level, by accelerated aging of collagen. Although CML and pentosidine are only two out of many, as yet unidentified, products of the Maillard reaction, they may be regarded as "biomarkers" of the overall extent of glycoxidative aging of proteins $(8)$ in both nondiabetic and diabetic populations.

Role of oxidative stress. In recent work from several laboratories $(8,20-24)$, oxidative stress and increased oxidative damage to biomolecules have been proposed as possible unifying mechanisms for the development of complications in diabetes. Although the level of glycoxidative damage to collagen is clearly increased in the diabetic patients (Fig. 7), an important question that can be asked from our data is whether the increased levels of glycoxidation products in collagen can be attributed solely to the increase in glycation, or whether an increase in oxidative stress is also involved. For this reason we have attempted to predict the levels of glycoxidation products and fluorescence in our diabetic patients, based on the age of onset and duration of diabetes, and the relative increase in glycemia. The assumption is made that Maillard products accumulated in collagen at a "normal" rate until the onset of diabetes, and thereafter at an accelerated rate, the difference being directly proportional to the increase in glycation of protein, either collagen or hemoglobin. The age of onset and duration of diabetes were known for each patient, and we used each patient's relative increase in glycation of either collagen or hemoglobin, compared to age-matched controls, at the time of biopsy as an indicator of long-term glycemic control. The following formula was used for the calculations:

$P L=C R[A O+(R G \times D)]$,

where $P L=$ predicted level of glycoxidation product or fluorescence; $C R=$ control rate of accumulation (per year) of glycoxidation product or fluorescence in collagen, i.e., slope of control (lower) line in Figs. 4-6; $A O=$ age of onset of diabetes (years); $R G=$ ratio of glycation of collagen or hemoglobin $\left(\% \mathrm{HbA}_{1}\right)$ in 
the patient, compared with age-matched nondiabetic control subject; $D=$ duration of diabetes (years).

From these calculations, ratios of observed (actual) to predicted values for each parameter were calculated. As shown in Table I, the observed levels of each glycoxidation product and fluorescence in diabetic skin collagen were consistently lower than predicted. The underestimate was more marked when based on the relative increase in glycation of collagen than hemoglobin in diabetic patients. Also, the underestimate remained if mean $\mathrm{HbA}_{1}$ values (calculated from results obtained every 3 mo over a 6 -yr period preceding the skin biopsy) were used in the calculation. Thus, in all cases, observed levels were lower than would be expected. We conclude that the increased accumulation of glycoxidation products and fluorescence in diabetic skin collagen can be explained, on average, solely by the increase in glycation for the duration of disease: we have found no evidence of a general increase in oxidative stress in diabetes. Indeed, the lower than expected levels of glycoxidation products and fluorescence, i.e., the ratios of $<1.0$ in Table I, suggest that oxidative stress may actually be decreased, on average, in diabetic patients. These analyses assume that $\mathrm{CML}$, pentosidine, and fluorescence are derived predominantly from interactions between proteins and glucose (i.e., as opposed to ribose or ascorbate), and that the increase in glycoxidation products is related to the increase in average glycemia, as measured by the increase in glycation of proteins. The conclusions are valid unless one assumes that the rate of formation of $\mathrm{CML}$, pentosidine, and increased fluorescence in collagen is primarily determined by factors other than the extent of glycation of proteins, e.g., variations in oxidative stress and/or levels of carbohydrates other than glucose. Although this hypothesis cannot be excluded, and although a possible role for increased oxidative stress has recently been emphasized by Wolff et al. (23), we have found a strong correlation between levels of FL and CML in urine from diabetic patients, suggesting a relationship between glycation of protein and the formation of CML (25). Notably, in that study, we observed that the ratio of CML to FL was decreased in urine from diabetic, compared with nondiabetic, subjects. Again, this is consistent with a decrease in oxidative stress in diabetic subjects. In relation to the possibility that CML is derived to a significant extent from sources other than glycated proteins, recent (unpublished) findings in our laboratory have shown that plasma pentose levels are not

Table I. Ratio of Observed to Predicted Concentrations of Glycoxidation Products and Fluorescence in Skin Collagen from Diabetic Patients

\begin{tabular}{lcc}
\hline & \multicolumn{2}{c}{ Basis of calculation* } \\
\cline { 2 - 3 } Measurement & $\begin{array}{c}\text { Glycation of collagen } \\
\text { ratio* }\end{array}$ & $\begin{array}{c}\text { Glycation of hemoglobin } \\
\text { ratio* }\end{array}$ \\
\hline CML & $0.74 \pm 0.32$ & $0.82 \pm 0.37$ \\
Pentosidine & $0.65 \pm 0.41$ & $0.79 \pm 0.50$ \\
Fluorescence & $0.60 \pm 0.33$ & $0.73 \pm 0.38$
\end{tabular}

\footnotetext{
* Ratios of observed to predicted concentrations of glycoxidation products and fluorescence. The predicted values are calculated as described by Eq. 1 (see Discussion), using the relative increase in glycation of collagen (FL) (left column) or hemoglobin $\left(\mathrm{HbA}_{1}\right)$ (right column) for individual patients (mean $\pm \mathrm{SD}$ ).
}

increased in type 1 diabetic patients, and that in nondiabetic guinea pigs, ascorbate supplementation does not result in increased collagen FL, CML, or pentosidine content.

Regardless of the status of oxidative stress in diabetes, it is clear that the net glycoxidative modification of collagen is increased. It is also evident from the large standard deviations shown in Table I that the ratios, based on either glycation of collagen or hemoglobin, are not normally distributed about the mean. This implies that, although the average ratio of observed to predicted values is $<1.0$, some patients have levels of glycoxidation products and fluorescence which are much higher than would be predicted from duration of diabetes and severity of hyperglycemia. The presence of higher than average oxidative stress in these patients may be an inherent characteristic, unrelated to the presence of diabetes. Indeed, a considerable variation in the rates of accumulation of glycoxidation products with age, despite similar glycemia, is seen in the nondiabetic population (Figs. 4-6) . Diabetic individuals who are also under high oxidative stress and are therefore rapid accumulators of glycoxidation products may be particularly vulnerable to the development of complications. The association between levels of skin collagen glycoxidation products and complication status is discussed further in the following article.

\section{Acknowledgments}

The authors wish to thank Professor D. R. Hadden, Dr. L. Kennedy, and Dr. A. B. Atkinson (Royal Victoria Hospital), and Drs. D. A. J. Keegan, F. A. O'Connor, and W. E. Parkes (Altnagelvin Hospital) for permission to study patients under their care. We are grateful to the staff of the Biochemistry and Hematology Departments at the Royal Victoria and Altnagelvin Hospitals, to Drs. D. J. Pettitt and R. L. Hanson for statistical advice, and to Drs. D. J. Pettitt and P. H. Bennett for helpful discussions.

This work was supported in part by research grant DK-19971 from the National Institute of Diabetes, Digestive and Kidney Diseases (USA).

\section{References}

1. Cohen, M. P. 1986. Diabetes and Protein Glycation. Springer-Verlag, Inc. New York. 140 pp.

2. Dunn, J. A., J. S. Patrick, S. R. Thorpe, and J. W. Baynes. 1989. Oxidation of glycated proteins: age-dependent accumulation of $N^{*}$-(carboxymethyl)lysine in lens proteins. Biochemistry. 28:9464-9468.

3. Dunn, J. A., D. R. McCance, S. R. Thorpe, T. J. Lyons, and J. W. Baynes. 1991. Age-dependent accumulation of $N^{*}$-(carboxymethyl) lysine and $N^{*}$-(carboxymethyl)hydroxylysine in human skin collagen. Biochemistry. 30:12051210 .

4. Dyer, D. G., J. A. Blackledge, B. M. Katz, C. J. Hull, H. D. Adkisson, S. R. Thorpe, T. J. Lyons, and J. W. Baynes. 1991. The Maillard reaction in vivo. $Z$. Ernährungswiss. 30:29-45.

5. Sell, D. R., and V. M. Monnier. 1989. Structure elucidation of a senescence cross-link from human extracellular matrix: implication of pentoses in the aging process. J. Biol. Chem. 264:21597-21622.

6. Grandhee, S. K., and V. M. Monnier. 1991. Mechanism of formation of the Maillard protein cross-link, pentosidine: glucose, fructose and ascorbate as pentosidine precursors. J. Biol. Chem. 266:11649-11653.

7. Dyer, D. G., J. A. Blackledge, S. R. Thorpe, and J. W. Baynes. 1991. Formation of pentosidine during nonenzymatic browning of protein by glucose: identification of glucose and other carbohydrates as possible precursors of pentosidine in vivo. J. Biol. Chem. 266:11654-11660.

8. Baynes, J. W. 1991. Perspectives in diabetes: role of oxidative stress in development of complications in diabetes. Diabetes. 40:405-412.

9. Dunn, J. A., M. U. Ahmed, M. H. Murtiashaw, J. M. Richardson, M. D. Walla, S. R. Thorpe, and J. W. Baynes. 1990. Reaction of ascorbate with lysine and protein under autoxidizing conditions: formation of $N^{*}$-(carboxymethyl)lysine by reaction between lysine and products of autoxidation of ascorbate. Biochemistry. 29:10964-10970. 
10. Monnier, V. M., R. R. Kohn, and A. Cerami. 1984. Accelerated age-related browning of human collagen in diabetes mellitus. Proc. Natl. Acad. Sci. USA. 81:583-587.

11. Sell, D. R., and V. M. Monnier. 1990. End-stage renal disease and diabetes catalyze the formation of a pentose-derived crosslink from aging human collagen. J. Clin. Invest. 75:380-384.

12. McCance, D. R., D. G. Dyer, K. E. Bailie, S. R. Thorpe, J. W. Baynes, and T. J. Lyons. 1993. Maillard reaction products and their relation to complications in insulin dependent diabetes mellitus. J. Clin. Invest. 91:2470-2478.

13. Kennedy, J. H. 1984. Analytical Chemistry. Harcourt Brace Jovanovich, New York. 392-395.

14. Stegemann, H., and K. Stalder. 1967. Determination of hydroxyproline. Clin. Chim. Acta. 18:267-273.

15. Maekawa, T., T. K. Rathinasamy, K. I. Altman, and W. F. Forbes. 1970 Changes in collagen with age: the extraction of acid soluble skin collagen from the skin of mice. Exp. Gerontol. 5:177-186.

16. Menard, L., M. E. Dempsey, L. A. Blankenstein, H. Aleyassine, M Wacks, and J. S. Soeldner. 1980. Quantitative determination of glycosylated hemoglobin A, by agar gel electrophoresis. Clin. Chem. 26:1598-1602.

17. Lyons, T. J., and L. Kennedy. 1985. Nonenzymatic glycosylation of skin collagen in patients with limited joint mobility. Diabetologia. 28:2-5.

18. Vishwanath, V., K. E. Frank, C. A. Elmets, P. J. Dauchot, and V. M.
Monnier. 1986. Glycosylation of skin collagen in type I diabetes mellitus: correlations with long-term complications. Diabetes. 35:916-921.

19. Lyons, T. J., K. E. Bailie, D. G. Dyer, J. A. Dunn, and J. W. Baynes. 1991. Decrease in skin collagen glycation with improved glycemic control in patients with insulin dependent diabetes mellitus. J. Clin. Invest. 87:1910-1915.

20. Wolff, S. P. 1987. The potential role of oxidative stress in diabetes and its complications: novel implications for theory and therapy. In Diabetic Complications. M. J. C. Crabbe, editor. Churchill Livingstone, New York. 167-220.

21. Godin, D. V., and S. A. Wohaieb. 1988. Reactive oxygen radical processes in diabetes. In Oxygen Radicals in the Pathophysiology of Heart Disease. P. K. Singal, editor. Kluwer Academic Publishers, Boston. 303-322.

22. Oberley, L. W. 1988. Free radicals and diabetes. Free Radical Biol. Med. 5:113-124.

23. Wolff, S. P., Z. Y. Jiang, and J. V. Hunt. 1991. Protein glycation and oxidative stress in diabetes mellitus and ageing. Free Radical Biol. Med. 10:339352.

24. Vander Jagt, D. L., B. Robinson, K. K. Taylor, and L. A. Hunsaker. 1992. Reduction of trioses by NADPH-dependent aldo-keto reductases: aldose reductase, methylglyoxal, and diabetic complications. J. Biol. Chem. 267:4364-4369.

25. Knecht, K. J., J. A. Dunn, K. F. McFarland, D. R. McCance, T. J. Lyons, S. R. Thorpe, and J. W. Baynes. 1991. Effect of diabetes and aging on carboxymethyllysine levels in human urine. Diabetes. 40:190-196. 